\title{
Economic costs and preference-based health- related quality of life outcomes associated with childhood psychiatric disorders
}

\author{
Stavros Petrou, Samantha Johnson, Dieter Wolke, Chris Hollis, Puja Kochhar and Neil Marlow
}

\section{Background}

Childhood psychiatric disorders may have deleterious consequences through childhood and into adulthood.

\section{Aims \\ To estimate costs and preference-based health-related quality of life outcomes (health utilities) associated with a broad range of childhood psychiatric disorders during the eleventh year of life.}

\section{Method}

Participants in a whole-population study of extremely preterm children and term-born controls (EPICure) undertook psychiatric assessment using the Development and Well Being Assessment (DAWBA) and the Kaufman-Assessment Battery for Children. Questionnaires completed by parents and teachers described the children's utilisation of health, social and education services during the eleventh year of life. Parents also described their child's health status using the Health Utilities Index Mark 2 and Mark 3 health status classification systems. Descriptive and multiple regression techniques were used to explore the association between psychiatric disorders and economic outcomes.

\section{Results}

The study presents detailed costs and health utilities associated with psychiatric disorders for the preterm population, term-born population and pooled study population, following appropriate controls.

\section{Conclusions}

The results of this study should be used to inform future economic evaluations of interventions aimed at preventing childhood psychiatric disorders or alleviating their effects. Further research is required that identifies, measures and values the longer-term economic impacts of these disorders in a valid and reliable manner.

\section{Declaration of interest}

None.
The median prevalence of childhood psychiatric disorders has been reported as $12 \%$ for prepubescent school-age children and $15 \%$ among adolescents. ${ }^{1}$ Among British children aged 5-15 years, it has been estimated that $10 \%$ have a psychiatric disorder, with $5 \%$ having conduct disorder and $4 \%$ having emotional disorders. ${ }^{2}$ A longitudinal community study of children aged 9-13 years from the American state of North Carolina estimated that the 3-month prevalence of any psychiatric disorder averaged $13.3 \%$ (95\% CI $11.7-15.0 \%)$. $^{3}$ There is increasing evidence from longitudinal studies ${ }^{4-14}$ and retrospective reports ${ }^{15}$ that childhood psychiatric disorders may have significant adverse consequences for mental health, educational outcomes and substance misuse through childhood and into adulthood. However, relatively little is known about their consequences in terms of costs or health utilities (preference-based measures of health outcome developed from economic theory), which can directly or indirectly inform resource-allocation decisions. Cost of illness studies have been conducted for a relatively small number of childhood psychiatric disorders, including childhood depression, ${ }^{16}$ separation anxiety ${ }^{17}$ and attention-deficit hyperactivity disorder (ADHD), ${ }^{18}$ and health utilities have been estimated for $\mathrm{ADHD}^{19,20}$ and autism-spectrum disorders. ${ }^{20}$ The purpose of this research is to augment the limited economic evidence in this area by estimating costs and health utilities associated with childhood psychiatric disorders during midchildhood. This will provide a significant new resource for clinical decision-makers and budgetary and service planners and to analysts estimating the cost-effectiveness of preventive or treatment interventions for these disorders.

\section{Method}

\section{Study population}

Children who participated in the EPICure study represented the study population for this empirical investigation. The EPICure study is a whole-population longitudinal study of all infants born at 20 to 25 completed weeks of gestation in all 276 maternity units in the UK and Republic of Ireland from March to December $1995 .{ }^{21}$ Participants in the EPICure study were selected for this investigation as previous research had indicated higher prevalence rates of psychiatric disorders among children born preterm or with a low birth weight compared with general population samples. ${ }^{22-25}$ Of the 307 surviving extremely preterm infants in the EPICure study, $241(78.2 \%)$ were assessed at a median age of 6 years 4 months (range: 5 years 2 months to 7 years 3 months $)^{26}$ and $219(71.3 \%)$ again at a median age of 10 years 11 months (range: 10 years 1 month to 12 years 1 month). ${ }^{27} \mathrm{~A}$ control group of 153 mainstream school classmates who were born at full term and matched for age, gender and ethnic group was also evaluated at a median age of 10 years 11 months (range: 9 years 9 months to 12 years 3 months). A full description of the EPICure study is available elsewhere. ${ }^{21,27}$ The extremely preterm children and their classmate controls were analysed separately for the purposes of the empirical investigation reported in this paper. Additionally, we analysed the pooled study population controlled for clinical and sociodemographic confounders, including gestational age at birth and a measure of neurosensory or motor impairment. Ethical approval for the study was obtained 
from the Southampton and South West Hampshire Research Ethics Committee and approved by the Central Office for Research Ethics Committees (COREC).

\section{Psychiatric assessments}

Childhood psychiatric disorders were diagnosed using the Development and Well Being Assessment (DAWBA), ${ }^{28}$ a semistructured interview conducted with the main parent (usually the mother) or completed online by the main parent around the child's eleventh birthday. Information obtained from the DAWBA was used to assign ICD-10 ${ }^{29}$ and DSM-IV-TR ${ }^{30}$ diagnoses. Supplemental information was provided by teachers who completed a questionnaire-based version of the DAWBA. Multiinformant data were collated and potential diagnoses were computer-generated using scoring algorithms. These were reviewed by two child and adolescent psychiatrists (C.H. and P.K.) masked to group allocation who assigned final DSM-IV and ICD-10 consensus diagnoses by reviewing quantitative symptom data and qualitative transcripts. In this paper we primarily refer to psychiatric disorders defined using DSM-IV-TR criteria. ${ }^{31}$ Diagnostic classifications were assigned to the categories of emotional, ADHD, conduct, autistic and tic disorders. In addition, the Kaufman-Assessment Battery for Children (K-ABC) was used to obtain IQ scores (range $40-160) .{ }^{32}$ Cognitive impairment was defined as either moderate $(-3$ standard deviations to -2 standard deviations or IQ scores of 71 to 81$)$ or severe $(<-3$ standard deviations or IQ scores of $\leqslant 70$ ) using the mean (s.d.) of classmates to account for the secular drift in IQ scores over time. ${ }^{27,33}$

\section{Estimation of costs}

As part of the battery of assessments performed at 11 years, the main parent was asked to complete a detailed postal questionnaire about their child's resource utilisation over the previous year of life. The questionnaire was piloted to ascertain its acceptability, comprehension and reliability and reminder letters were sent to parents to increase the response and completion rates. The data collected from the main parent included their child's use of hospital in-patient and day care services, community health services, prescribed medications, social services and education services. The components of resource utilisation and the units in which they were measured are summarised in online Table DS1. Estimates of service provision were derived from these data and usually expressed in terms of contact hours. For all hospital admissions, estimates of service provision were expressed in terms of patient days with part of a day at each level of care counted as a 24-hour period. For education services, estimates of service provision reflected the level of educational assistance within each type of educational establishment (mainstream school, mainstream school with special unit attached, special school for the physically disabled and special school for children with intellectual disability or learning difficulties). In addition to information provided by parents, teachers were asked to identify children with special educational needs, defined in the educational context as those with intellectual disability or learning difficulties that make it harder for them to learn or access education than most children of the same age, and they were also asked to detail any special educational needs support the child received. ${ }^{27}$ This included information on the type and duration of individual education or behavioural plans, one-to-one special needs provision, small group special needs provision, outreach support and support from educational psychologists, clinical psychologists, physiotherapists, speech therapists and occupational therapists. All resource-use data were entered directly from the research instruments into a purpose-built data-collection program with in-built safeguards against inconsistent entries and then verified by dual coding.

United Kingdom unit costs were applied to each resource item to value total resource use for each study child over an annual period. All unit costs employed followed recent guidelines on costing public services as part of economic evaluation. ${ }^{34-36}$ The calculation of these costs was underpinned by the concept of opportunity cost, which can be defined as the value of the next best alternative for using these resources. ${ }^{35}$ The costs of hospital in-patient and day care services were largely derived from English Department of Health reference costs based upon National Health Service trust financial returns. ${ }^{37}$ The unit costs of community health and social services were largely derived from national sources, ${ }^{38}$ and took account of time spent by professionals on indirect activities, such as travelling and paper work. Some unit costs of health and social services were calculated from first principles using established accounting methods. ${ }^{34}$ Drug costs were obtained from the British National Formulary. ${ }^{39}$ Educational costs were based upon data for different types of educational establishment obtained from the Department of Education and Skills in England (details available from the author on request). These included salaries, employer on-costs and revenue and capital overheads associated with each form of special educational needs support described above. All costs were expressed in pounds sterling and reflected values for the financial year 2006-7.

\section{Estimation of health utilities}

The postal questionnaire completed by the main parent around the child's eleventh birthday included the Health Utilities Index (HUI), which can be described as a family of health status classification systems with preference weights (or multi-attribute utility scores) attached to each permutation of responses. ${ }^{40}$ The main parent was considered the appropriate subject for completing the HUI as related research had indicated that the comprehension level required for successful completion is somewhat higher for a paediatric sample where a number of children have developmental disabilities. ${ }^{41}$ The main parent completed the unedited 15-item questionnaire for proxy-assessed usual health status assessment, which was obtained from the HUI developers and covers both Mark 2 (HUI2) and Mark 3 (HUI3) health status classification systems. The 'usual' health focus of the questions has previously been applied in population health surveys, where short-term illnesses such as influenza are not the major concern. ${ }^{42}$ The HUI2 health status classification system covers seven attributes: sensation, mobility, emotion, cognition, self-care, pain and fertility. The HUI3 health status classification system covers eight attributes: cognition, vision, hearing, speech, ambulation, dexterity, emotion and pain. The HUI3 health status classification system is now recommended by the HUI developers as the preferred measure of primary analyses because of its broad applicability in both clinical and general population health studies, improvements in a number of definitions, and an increased orthogonality of its attributes for structural independence. $^{42,43}$ Consequently, our primarily analyses of preference-based health-related quality of life outcomes were based on the HUI3 health status classification system. Function within each HUI3 attribute is graded on a five- or six-point scale corresponding to the level of severity, ranging from normal function (level 1) to severe impairment (level 5 or 6). Responses to the HUI3 health status classification system were converted into multiplicative multi-attribute utility scores using a published utility function. ${ }^{44,45}$ These multi-attribute utility scores are based on the permutation of responses across the eight attributes and are 
expressed on an interval scale ranging from -0.36 (representing the health state with the lowest level of function for all attributes) to 1.00 (representing the health state with the highest level of function for all attributes). The multi-attribute utility scoring algorithm for the HUI3 can be summarised as

$$
u^{*}=1.371\left(b_{1} \times b_{2} \times b_{3} \times b_{4} \times b_{5} \times b_{6} \times b_{7} \times b_{8}\right)-0.371
$$

where $u^{*}$ is the utility score for the overall health state being measured and the $b_{j}$ 's are substituted from a table of coefficients provided by the HUI developers for the appropriate attribute and level. ${ }^{45}$ To develop the multi-attribute utility scoring algorithm a random sample of 504 general population adults living in the city of Hamilton, Canada had previously been asked to value selected health states using both a visual analogue scaling technique and a standard gamble instrument. ${ }^{45}$ Analyses of preference-based health-related quality of life outcomes in our study were repeated using the HUI2 health status classification system and an underpinning multi-attribute utility scoring algorithm recently estimated on the basis of the preferences of 198 members of the UK general population. ${ }^{46}$ The latter measure and underpinning multi-attribute utility scoring algorithm might be considered to generate relevant values for UK policy purposes. $^{36}$

\section{Statistical methods}

Differences in baseline sociodemographic and clinical characteristics between children with and without a psychiatric disorder, as defined by DSM-IV-TR criteria, were tested using the Pearson chi-squared test.

A number of statistical approaches were tested in order to impute costs for children with some missing data. Given the negligible level of missing cost data $(<2 \%)$ in the final study sample, simple linear regression and simulation-based multiple imputation ${ }^{47}$ for each psychiatric group produced similar results. Therefore, it was considered appropriate to use the estimates generated by the simple linear regression in this analysis. ${ }^{4-49}$

For the preterm population, term-born population and pooled study population, comparisons of each category of public sector costs and of total public sector costs were made between children with and without a psychiatric disorder as defined by DSM-IV-TR criteria. Similarly, for the preterm, term-born and pooled populations, comparisons of total public sector costs were made between children with and without varying levels of cognitive impairment. Comparisons of total public sector costs between children with and without individual psychiatric disorders (emotional, ADHD, conduct, autistic, tic) as defined by DSM-IV-TR criteria were restricted to the pooled population because of the relatively small sample sizes for some disorders (e.g. $n=4$ for tic disorders). Comparisons of costs are reported as mean values with standard deviations and mean differences in costs between the comparison groups with $95 \%$ confidence intervals. As the data for costs were skewed, in addition to Student $t$-tests of cost differences, non-parametric bootstrap estimation was used to derive $95 \%$ confidence intervals for mean cost differences between the comparison groups. ${ }^{50}$ The bootstrap method does not rely on parametric assumptions concerning the underlying distribution of data, hence its usefulness for generating confidence intervals for skewed data. ${ }^{51}$ Using a large number of simulations, and based on sampling with replacement from the original data, the bootstrap method estimates the distribution of a sampling statistic. $^{51}$ Each of the confidence intervals surrounding mean cost differences was calculated using 10000 bias-corrected bootstrap replications.
In addition, generalised linear regressions ${ }^{51}$ were performed for the preterm, term-born and pooled populations with total public sector costs over the previous year of life representing the dependent variable in the analyses. Three regression models were constructed for each population group. In the first model, the main independent variable was a dichotomous variable of whether or not the child had a psychiatric disorder as defined by DSM-IV-TR criteria. In the second model, the main independent variable was a dichotomous variable of whether or not the child had moderate cognitive impairment, whereas in the third model it was a dichotomous variable of whether or not the child had severe cognitive impairment. For each generalised linear regression model, a gamma distribution and linear (identity) link function for costs was selected on the basis of its low Akaike information criterion (AIC) statistic (AIC statistics of 19.24, 19.24 and 19.23 for models 1,2 and 3 respectively for the pooled population) compared with alternative distributional forms (e.g. Gaussian, inverse Gaussian and Poisson distributional families) and link functions (e.g. log link function). For the preterm and term-born populations, covariates included in the generalised linear regressions were gender (male, female), maternal marital status (married, single, cohabiting, widowed, separated or divorced), respondent parent's current age $(<30$, $30-39,40-49, \geqslant 50$ years), type of accommodation (owner occupied, rented, other), access to car (yes, no), highest parental qualification (vocational or equivalent, ordinary level or equivalent, advanced level or equivalent, diploma or equivalent, university degree, postgraduate qualification, other, none), highest parental occupational status (professional or managerial, intermediate, routine and manual, long-term unemployed), language spoken at home (English only, English and other language(s)), number of smokers at home $(0,1, \geqslant 2)$ and a measure of neurosensory or motor impairment (no, yes). ${ }^{52}$ For the pooled population, covariates includes in the generalised linear regressions additionally included gestational age at birth ( $\leqslant 23$ weeks, 24 weeks, 25 weeks, term). In sensitivity analyses, the measure of neurosensory or motor impairment was replaced by an interaction term between gestational age at birth and psychiatric disorder for the pooled population.

For the preterm population, term-born population and pooled study population, we used Fisher's exact test for equality of proportions to compare the proportion of children with suboptimal levels of function (defined as below level 1 function) within each of the eight attributes of the HUI3 health status classification system between children with and without a psychiatric disorder as defined by DSM-IV-TR criteria. The same comparison groups used as a basis for analysing total public sector costs were also used as a basis for analysing health utilities. Differences in the HUI3 and UK HUI2 multi-attribute utility scores between the comparison groups were tested using twosample $t$-tests for unequal variance. Finally, we performed Tobit regressions to explore the effects of psychiatric disorders on the HUI3 and UK HUI2 multi-attribute utility scores for the preterm, term-born and pooled populations. Tobit regression was required to account for the censoring of the dependent variable, the multiattribute utility score, which has an upper value of 1.0. ${ }^{53}$ As with costs, three regression models were constructed for each population group, which differed in terms of the main psychiatric independent variable. The same covariates incorporated into the generalised linear regressions on costs were incorporated into the Tobit regressions on health utilities.

All analyses were performed with a microcomputer using the Statistical Package for the Social Sciences (SPSS) (version 15.0) software and STATA (version 10.0) software for Windows XP. $P$-values of 0.05 or less were considered statistically significant. 


\section{Results}

Postal questionnaires reporting costs and health utilities were returned for a total of 331 study children, including 190 extremely preterm children (representing $86.8 \%$ of extremely preterm children undergoing neurodevelopmental assessments at a median age of 10 years 11 months) and 141 term-born classmates (representing $92.2 \%$ of term-born classmates undergoing neurodevelopmental assessments at this time point). Multi-informant psychiatric assessments were performed on 321 (97.0\%) of these 331 study children, with multiple imputation techniques used to estimate psychiatric diagnoses for the remaining 10 children. $^{25}$ Children for whom postal questionnaires were not returned were more likely to be born at between 25 weeks exactly and 25 weeks 6 days, be of Black and minority ethnic origin, have had an operation for necrotising enterocolitis, to have unemployed parents and to have had lower cognitive scores or cognitive impairment at 2.5 and 6 years $(P \geqslant 0.05)$. There were a number of significant differences between the 50 children with and 281 without a DSM-IV-TR clinical diagnosis, for whom postal questionnaires were returned, in terms of sociodemographic and clinical characteristics. Notably, children with a DSM-IV-TR clinical diagnosis were more likely to be male, less likely to have married parents, less likely to live in owner-occupied accommodation, more likely to live with smokers and more likely to have been born preterm (online Table DS2).

Resource-use values for children with and without a psychiatric disorder as defined by DSM-IV-TR criteria are summarised in online Table DS1 for the pooled study population. This table highlights significant differences in mean utilisation of a number of mental and non-mental health and social care services between these groups.

Public sector costs over the previous year of life for the preterm population, term-born population and pooled study population are summarised in online Table DS3. Among the pooled study population, mean (s.d.) public sector costs over the 12 -month period were $\mathfrak{E 7 1 8 8}$ (s.d. $=£ 5869$ ) for the 50 children with a psychiatric disorder, as defined by DSM-IV-TR

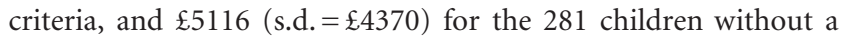
psychiatric disorder, generating a mean cost difference of $£ 2072$ (bootstrap 95\% CI $£ 349-£ 3795$ ), which was statistically significant $(P=0.020)$. Among the preterm population, the mean public sector cost difference between the 39 children with and 151 children without a psychiatric disorder, as defined by DSM-IV-TR criteria, was estimated at $£ 1998$ (bootstrap

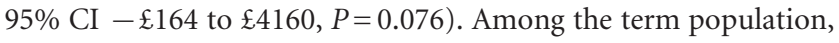
the respective public sector cost difference between the 11 children with and 130 children without a psychiatric disorder, as defined by DSM-IV-TR criteria, was estimated at $\mathfrak{E} 51$ (bootstrap 95\% CI

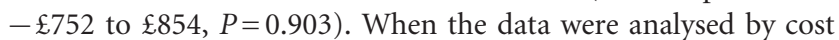
category, a DSM-IV-TR clinical diagnosis was associated with significantly higher community health and social care costs in all population groups and significantly higher total health and social care costs in the preterm population and pooled study population.

Mean public sector costs over the previous year of life and mean cost differences between children with and without individual psychiatric disorders are summarised in Table 1 for the preterm population, term-born population and pooled study population. Of particular note are the additional $£ 3170$ $(P=0.001)$ and $£ 8877(P<0.0001)$ annual public sector costs associated with moderate cognitive impairment and severe cognitive impairment, respectively, in the preterm population; and the additional $\mathfrak{E} 6745(P=0.014), \mathfrak{E} 3375(P<0.0001)$ and $\mathfrak{E} 8530$ $(P<0.0001)$ annual public sector costs associated with a diagnosis of an autistic disorder, moderate cognitive impairment and severe cognitive impairment, respectively, in the pooled study population.

The results of generalised linear regressions exploring the relationship between psychiatric disorders and total public sector

\begin{tabular}{|c|c|c|c|c|c|c|c|c|c|}
\hline \multirow[b]{2}{*}{ Psychiatric disorder } & \multicolumn{3}{|c|}{ With disorder } & \multicolumn{3}{|c|}{ Without disorder } & \multicolumn{3}{|c|}{ Cost difference } \\
\hline & $n$ & Mean & s.d. & $n$ & Mean & s.d. & Mean & $95 \% \mathrm{Cl}^{\mathrm{a}}$ & $P^{b}$ \\
\hline \multicolumn{10}{|l|}{ Preterm sample } \\
\hline Any DSM-IV clinical diagnosis & 39 & 8071.8 & 6358.5 & 151 & 6073.8 & 5264.9 & 1998.0 & -164.4 to 4160.4 & 0.076 \\
\hline Moderate cognitive impairment ${ }^{c}$ & 67 & 8536.1 & 6665.3 & 123 & 5366.0 & 4481.2 & 3170.0 & 1384.4 to 4955.7 & 0.001 \\
\hline Severe cognitive impairment ${ }^{d}$ & 18 & 14519.4 & 6187.6 & 172 & 5643.0 & 4765.3 & 8876.5 & 5923.5 to 11829.5 & $<0.0001$ \\
\hline \multicolumn{10}{|l|}{ Term sample } \\
\hline Any DSM-IV clinical diagnosis & 11 & 4053.9 & 1127.7 & 130 & 4003.3 & 2624.7 & 50.6 & -752.3 to 853.6 & 0.903 \\
\hline Moderate cognitive impairment $^{\mathrm{C}}$ & 2 & 3333.0 & 121.6 & 139 & 4017.0 & 2554.5 & e & e & e \\
\hline Severe cognitive impairment ${ }^{d}$ & 0 & - & - & 141 & 4007.3 & 2537.5 & e & e & e \\
\hline \multicolumn{10}{|l|}{ Total sample } \\
\hline Any emotional disorder ${ }^{f}$ & 16 & 6860.1 & 5259.2 & 315 & 5433.4 & 4738.9 & 1426.8 & -1195.1 to 4048.7 & 0.304 \\
\hline Any ADHD diagnosisg & 17 & 5812.0 & 3832.6 & 314 & 5551.1 & 4852.0 & 261.0 & -1656.8 to 2178.7 & 0.792 \\
\hline Any conduct disorder ${ }^{\mathrm{h}}$ & 17 & 7033.5 & 5700.1 & 314 & 5342.0 & 4609.3 & 1691.5 & -1006.2 to 4389.2 & 0.246 \\
\hline Any autistic disorder ${ }^{i}$ & 11 & 12016.1 & 7568.1 & 320 & 5270.8 & 4481.2 & 6745.3 & 2232.9 to 11257.7 & 0.014 \\
\hline Tic disorder & 4 & 7022.4 & 6474.7 & 327 & 5482.7 & 4760.2 & 1539.7 & -4815.1 to 7894.5 & 0.667 \\
\hline Any DSM-IV clinical diagnosis & 50 & 7187.8 & 5868.6 & 281 & 5115.9 & 4369.5 & 2071.9 & 348.7 to 3795.2 & 0.020 \\
\hline Moderate cognitive impairment $^{\mathrm{C}}$ & 69 & 8385.3 & 6625.2 & 262 & 4650.3 & 3645.8 & 3735.0 & 2087.8 to 5382.1 & $<0.0001$ \\
\hline Severe cognitive impairment ${ }^{d}$ & 18 & 13443.3 & 6725.1 & 313 & 4913.5 & 4011.8 & 8529.8 & 5554.9 to 11504.7 & $<0.0001$ \\
\hline \multicolumn{10}{|c|}{$\begin{array}{l}\text { ADHD, attention-deficit hyperactivity disorder. } \\
\text { a. Bootstrap estimation using } 10000 \text { replications, bias corrected. } \\
\text { b. P calculated using Student's } t \text {-test. } \\
\text { c. IQ score: }-3 \text { standard deviations to }-2 \text { standard deviations against classmate reference norms. } \\
\text { d. IQ score: Iess than }-3 \text { standard deviations against classmate reference norms. } \\
\text { e. Not calculated because of insufficient cases. } \\
\text { f. Encompasses separation anxiety, specific phobia, social phobia, post-traumatic stress disorder, generalised anxiety disorder, childhood emotional disorder (not otherwise specified) } \\
\text { and major depression. } \\
\text { g. Encompasses ADHD inattentive subtype and ADHD combined subtype. } \\
\text { h. Encompasses conduct disorder and oppositional defiant disorder. } \\
\text { i. Encompasses childhood autism and atypical autism. }\end{array}$} \\
\hline
\end{tabular}




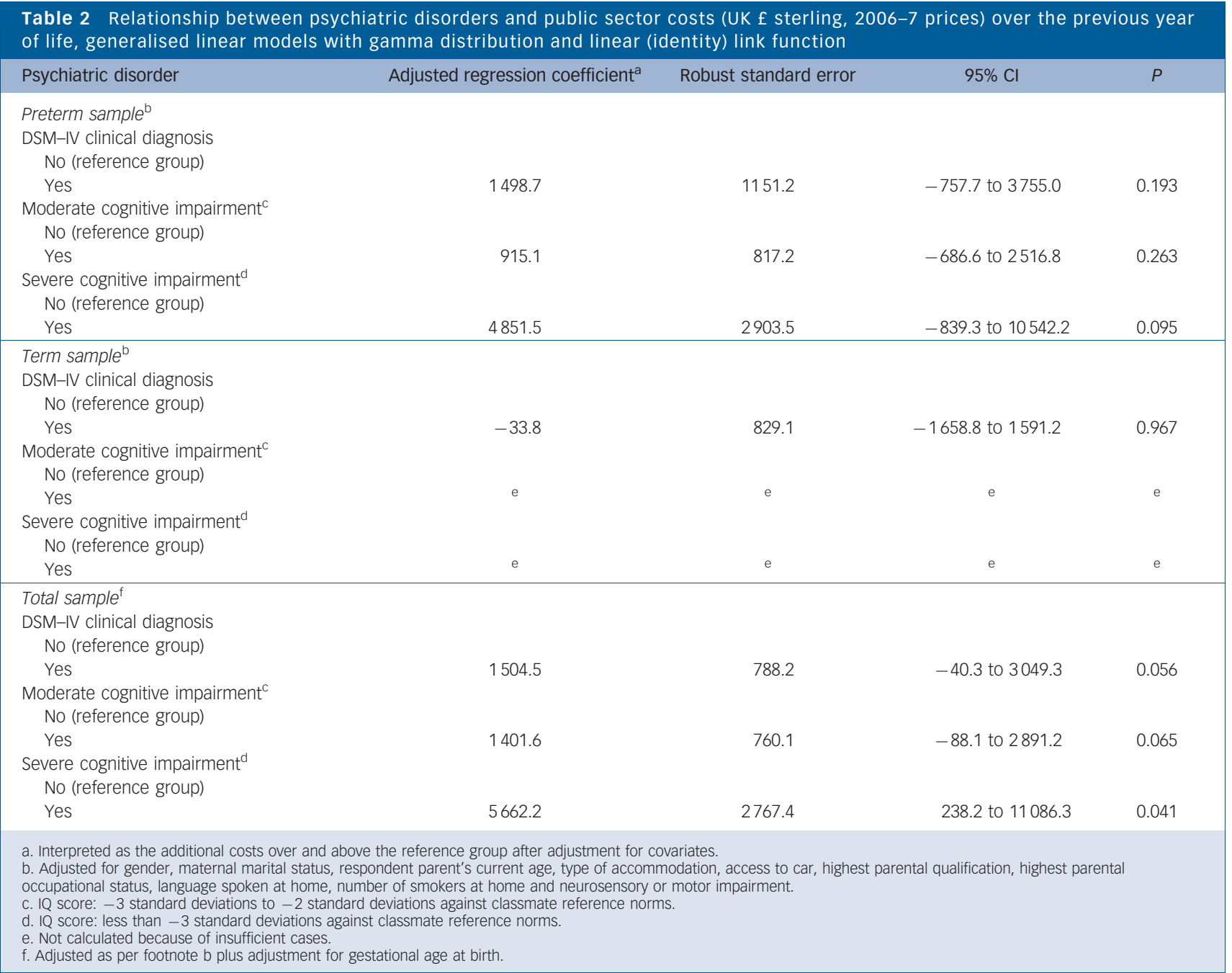

costs over the previous year of life are shown in Table 2 for the preterm population, term-born population and pooled study population. After controlling for clinical and sociodemographic confounders, a DSM-IV-TR clinical diagnosis was associated with

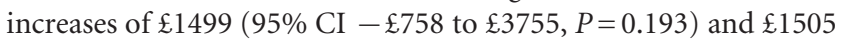

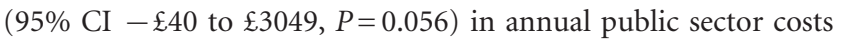
for the preterm and pooled study populations, respectively. Moderate cognitive impairment and severe cognitive impairment were associated with increases in annual public sector costs of $£ 915$ (95\% CI $-£ 687$ to $£ 2517, P=0.263$ ) and $£ 4852$ (95\% CI

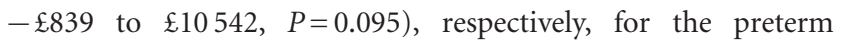

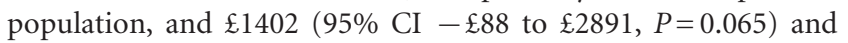

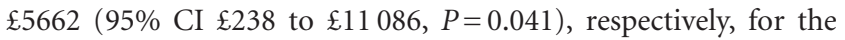
pooled study population. The only other factor associated with significantly increased public sector costs across the regression models was extremely preterm birth for the pooled study population. Replacing the measure of neurosensory or motor impairment ${ }^{52}$ by an interaction term between gestational age at birth and psychiatric disorder had no discernible effects on the results of the generalised linear regressions.

In each population group, comparisons of the frequency and proportion of suboptimal levels of function were made between the children with and without a DSM-IV-TR clinical diagnosis for each of the eight attributes of the HUI3. These analyses revealed significantly higher proportions of suboptimal levels of function among the children with a DSM-IV-TR clinical diagnosis for four attributes (emotion, pain, dexterity and cognition) for the preterm population, three attributes (speech, emotion and dexterity) for the term population and five attributes (speech, emotion, pain, dexterity and cognition) for the pooled study population $(P \leqslant 0.05)$.

Mean HUI3 multi-attribute utility scores and mean utility differences between children with and without individual psychiatric disorders are summarised in Table 3 for all three populations. Of particular note are the $0.165(P=0.003), 0.232$ $(P<0.0001)$ and $0.512 \quad(P<0.0001)$ mean utility decrements associated with a psychiatric disorder as defined by DSM-IV-TR criteria, moderate cognitive impairment and severe cognitive impairment, respectively, in the preterm population; and the $0.198 \quad(P=0.027), 0.250 \quad(P=0.003), 0.261 \quad(P=0.011), 0.192$ $(P<0.0001), 0.273 \quad(P<0.0001)$ and $0.571 \quad(P<0.0001)$ mean utility decrements associated with an emotional disorder, an ADHD diagnosis, an autistic disorder, a psychiatric disorder as defined by DSM-IV-TR criteria, moderate cognitive impairment and severe cognitive impairment, respectively, in the pooled study population. Analogous results were generated using the alternative UK HUI2 multi-attribute utility measure with the exception that statistically significant utility decrements were also generated for a psychiatric disorder as defined by DSM-IV-TR criteria for the term-born population and a conduct disorder for the pooled study population (Table 4).

Finally, the separate Tobit regressions revealed that, even after controlling for clinical and sociodemographic confounders, a psychiatric disorder as defined by DSM-IV-TR criteria, moderate 
Table 3 Health Utilities Index Mark 3 multi-attribute utility scores for children with and without psychiatric disorders

\begin{tabular}{|c|c|c|c|c|c|c|c|c|c|}
\hline \multirow[b]{2}{*}{ Psychiatric disorder } & \multicolumn{3}{|c|}{ With disorder } & \multicolumn{3}{|c|}{ Without disorder } & \multicolumn{3}{|c|}{ Cost difference } \\
\hline & $n$ & Mean & s.d. & $n$ & Mean & s.d. & Mean & $95 \% \mathrm{Cl}$ & $P^{\mathrm{a}}$ \\
\hline \multicolumn{10}{|l|}{ Preterm sample } \\
\hline Any DSM-IV clinical diagnosis & 39 & 0.656 & 0.270 & 151 & 0.820 & 0.254 & 0.165 & 0.061 to 0.269 & 0.003 \\
\hline Moderate cognitive impairment ${ }^{b}$ & 67 & 0.635 & 0.331 & 123 & 0.867 & 0.180 & 0.232 & 0.140 to 0.324 & $<0.0001$ \\
\hline Severe cognitive impairment ${ }^{\mathrm{C}}$ & 18 & 0.318 & 0.390 & 172 & 0.830 & 0.207 & 0.512 & 0.285 to 0.739 & $<0.0001$ \\
\hline \multicolumn{10}{|l|}{ Term sample } \\
\hline Any DSM-IV clinical diagnosis & 11 & 0.826 & 0.251 & 130 & 0.967 & 0.070 & 0.141 & -0.027 to 0.310 & 0.093 \\
\hline Moderate cognitive impairment ${ }^{b}$ & 2 & 0.884 & 0.165 & 139 & 0.957 & 0.102 & d & d & d \\
\hline Severe cognitive impairment ${ }^{c}$ & 0 & - & - & 141 & 0.956 & 0.102 & d & d & d \\
\hline \multicolumn{10}{|l|}{ Total sample } \\
\hline Any emotional disorder ${ }^{\mathrm{e}}$ & 16 & 0.672 & 0.296 & 315 & 0.871 & 0.220 & 0.198 & 0.026 to 0.371 & 0.027 \\
\hline Any ADHD diagnosis ${ }^{f}$ & 17 & 0.629 & 0.271 & 314 & 0.879 & 0.215 & 0.250 & 0.099 to 0.402 & 0.003 \\
\hline Any conduct disorderg & 17 & 0.727 & 0.260 & 314 & 0.870 & 0.221 & 0.143 & -0.008 to 0.294 & 0.062 \\
\hline Any autistic disorder ${ }^{\mathrm{h}}$ & 11 & 0.609 & 0.257 & 320 & 0.870 & 0.222 & 0.261 & 0.076 to 0.446 & 0.011 \\
\hline Tic disorder & 4 & 0.675 & 0.292 & 327 & 0.866 & 0.224 & 0.190 & -0.529 to 0.909 & 0.376 \\
\hline Any DSM-IV clinical diagnosis & 50 & 0.698 & 0.273 & 281 & 0.890 & 0.203 & 0.192 & 0.105 to 0.278 & $<0.0001$ \\
\hline Moderate cognitive impairment ${ }^{b}$ & 69 & 0.643 & 0.329 & 262 & 0.916 & 0.149 & 0.273 & 0.187 to 0.359 & $<0.0001$ \\
\hline Severe cognitive impairment ${ }^{c}$ & 18 & 0.318 & 0.390 & 313 & 0.889 & 0.178 & 0.571 & 0.345 to 0.797 & $<0.0001$ \\
\hline \multicolumn{10}{|c|}{$\begin{array}{l}\text { ADHD, attention-deficit hyperactivity disorder. } \\
\text { a. P calculated using Student's } t \text {-test. } \\
\text { b. IQ score: }-3 \text { standard deviations to }-2 \text { standard deviations against classmate reference norms. } \\
\text { c. IQ score: less than }-3 \text { standard deviations against classmate reference norms. } \\
\text { d. Not calculated because of insufficient cases. } \\
\text { e. Encompasses separation anxiety, specific phobia, social phobia, post-traumatic stress disorder, generalised anxiety disorder, childhood emotional disorder (not otherwise specified) } \\
\text { and major depression. } \\
\text { f. Encompasses ADHD inattentive subtype and ADHD combined subtype. } \\
\text { g. Encompasses conduct disorder and oppositional defiant disorder. } \\
\text { h. Encompasses childhood autism and atypical autism. }\end{array}$} \\
\hline
\end{tabular}

\begin{tabular}{|c|c|c|c|c|c|c|c|c|c|}
\hline \multirow[b]{2}{*}{ Psychiatric disorder } & \multicolumn{3}{|c|}{ With disorder } & \multicolumn{3}{|c|}{ Without disorder } & \multicolumn{3}{|c|}{ Cost difference } \\
\hline & $n$ & Mean & s.d. & $n$ & Mean & s.d. & Mean & $95 \% \mathrm{Cl}$ & $P^{a}$ \\
\hline \multicolumn{10}{|l|}{ Preterm sample } \\
\hline Any DSM-IV clinical diagnosis & 39 & 0.759 & 0.148 & 151 & 0.858 & 0.157 & 0.098 & 0.041 to 0.155 & 0.001 \\
\hline Moderate cognitive impairment ${ }^{b}$ & 67 & 0.754 & 0.186 & 123 & 0.883 & 0.123 & 0.130 & 0.077 to 0.182 & $<0.0001$ \\
\hline Severe cognitive impairment ${ }^{c}$ & 18 & 0.612 & 0.245 & 172 & 0.861 & 0.130 & 0.249 & 0.117 to 0.381 & 0.001 \\
\hline \multicolumn{10}{|l|}{ Term sample } \\
\hline Any DSM-IV clinical diagnosis & 11 & 0.854 & 0.131 & 130 & 0.948 & 0.077 & 0.094 & 0.005 to 0.183 & 0.040 \\
\hline Moderate cognitive impairment ${ }^{b}$ & 2 & 0.871 & 0.105 & 139 & 0.941 & 0.085 & d & d & d \\
\hline Severe cognitive impairment ${ }^{c}$ & 0 & - & - & 141 & 0.940 & 0.086 & d & d & d \\
\hline \multicolumn{10}{|l|}{ Total sample } \\
\hline Any emotional disorder ${ }^{\mathrm{e}}$ & 16 & 0.760 & 0.161 & 315 & 0.888 & 0.139 & 0.127 & 0.037 to 0.218 & 0.009 \\
\hline Any ADHD diagnosis ${ }^{f}$ & 17 & 0.792 & 0.120 & 314 & 0.888 & 0.142 & 0.096 & 0.028 to 0.164 & 0.008 \\
\hline Any conduct disorderg & 17 & 0.802 & 0.129 & 314 & 0.888 & 0.141 & 0.085 & 0.009 to 0.161 & 0.030 \\
\hline Any autistic disorder ${ }^{\mathrm{h}}$ & 11 & 0.721 & 0.152 & 320 & 0.887 & 0.140 & 0.165 & 0.056 to 0.275 & 0.007 \\
\hline Tic disorder & 4 & 0.801 & 0.156 & 327 & 0.884 & 0.141 & 0.083 & -0.164 to 0.329 & 0.367 \\
\hline Any DSM-IV clinical diagnosis & 50 & 0.782 & 0.149 & 281 & 0.901 & 0.133 & 0.118 & 0.071 to 0.165 & $<0.0001$ \\
\hline Moderate cognitive impairment ${ }^{b}$ & 69 & 0.757 & 0.185 & 262 & 0.915 & 0.108 & 0.158 & 0.109 to 0.206 & $<0.0001$ \\
\hline Severe cognitive impairment ${ }^{c}$ & 18 & 0.612 & 0.245 & 313 & 0.898 & 0.118 & 0.286 & 0.155 to 0.417 & $<0.0001$ \\
\hline \multicolumn{10}{|c|}{$\begin{array}{l}\text { ADHD, attention-deficit hyperactivity disorder. } \\
\text { a. P calculated using Student's } t \text {-test. } \\
\text { b. IQ score: }-3 \text { standard deviations to }-2 \text { standard deviations against classmate reference norms. } \\
\text { c. IQ score: less than }-3 \text { standard deviations against classmate reference norms. } \\
\text { d. Not calculated because of insufficient cases. } \\
\text { e. Encompasses separation anxiety, specific phobia, social phobia, post-traumatic stress disorder, generalised anxiety disorder, childhood emotional disorder (not otherwise specified) } \\
\text { and major depression. } \\
\text { f. Encompasses ADHD inattentive subtype and ADHD combined subtype. } \\
\text { g. Encompasses conduct disorder and oppositional defiant disorder. } \\
\text { h. Encompasses childhood autism and atypical autism. }\end{array}$} \\
\hline
\end{tabular}

cognitive impairment and severe cognitive impairment were associated with significant decrements in the HUI3 multi-attribute utility score of $0.226(P<0.0001), 0.205(P<0.0001)$ and 0.342 $(P<0.0001)$, respectively, for the preterm population, and 0.213 $(P<0.0001), \quad 0.198 \quad(P<0.0001)$ and $0.324 \quad(P<0.0001)$, respectively, for the pooled study population (Table 5$)$. Analogous results were generated using the alternative UK HUI2 multiattribute utility measure (Table 6); the decrements in these utility scores were smaller in magnitude, but remained statistically significant. The only other factor associated with statistically significant decrements in utility scores across the regression models was extremely preterm birth for the pooled study 


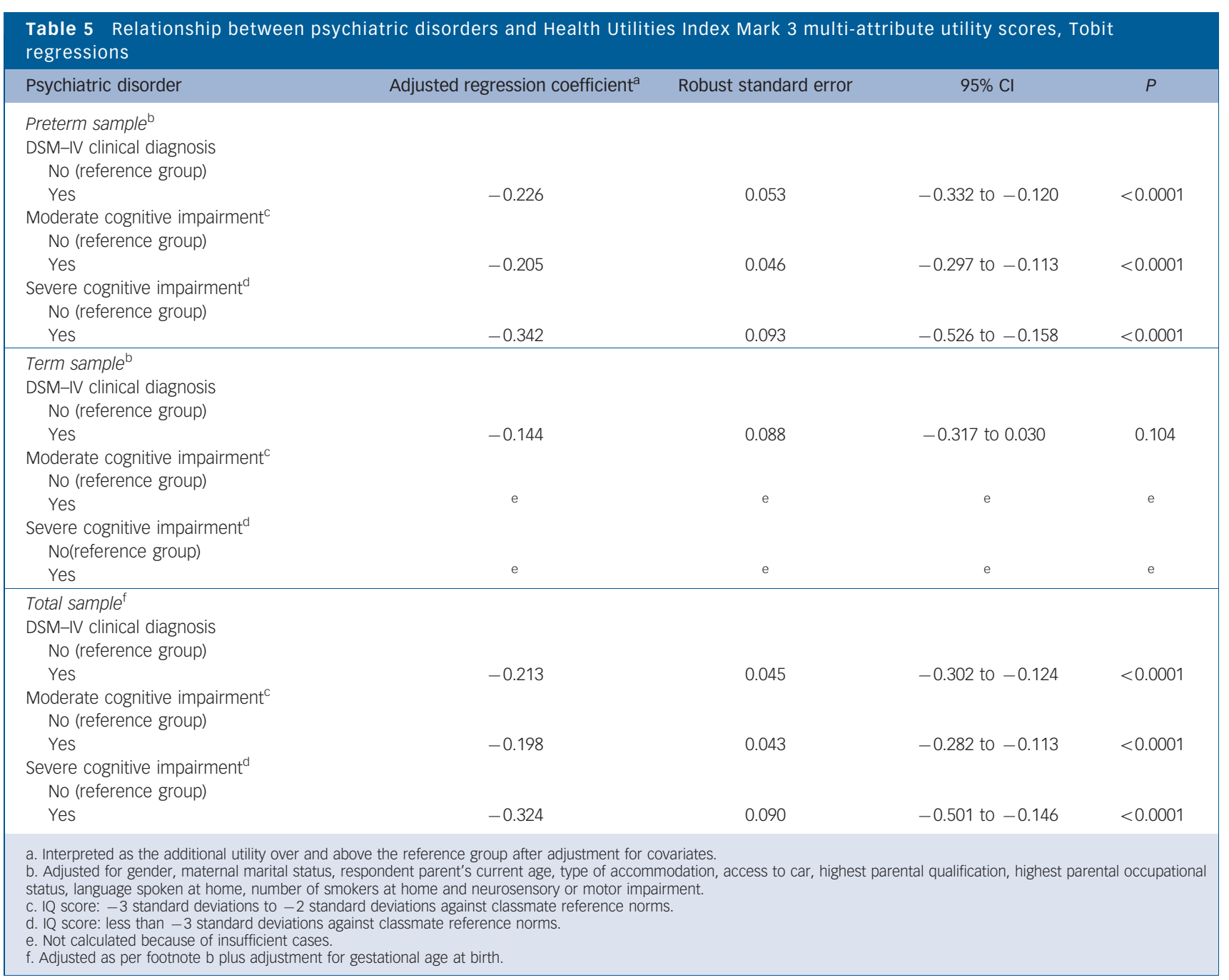

population. Replacing the measure of neurosensory or motor impairment ${ }^{53}$ by an interaction term between gestational age at birth and psychiatric disorder had no discernible effects on the results of the Tobit regressions.

\section{Discussion}

\section{Main findings}

This paper augments the limited published evidence on the economic consequences of childhood psychiatric disorders. ${ }^{16-20}$ Its unique contribution is twofold. First, it focuses on a broader range of childhood psychiatric disorders than has hitherto been studied by health economists within one sample, from relatively rare tic disorders to more common emotional and behavioural disorders, such as ADHD. Second, it reports both cost and preference-based health-related quality of life (or health utility) outcomes for these disorders. In the process, it provides a broader set of data inputs for directly or indirectly informing resource-allocation decisions than has hitherto been provided.

The study revealed an average annual cost difference of over $£ 2000$ across the pooled study population between children with and without a psychiatric disorder as defined by DSM-IV-TR criteria. This exceeds that identified for several other childhood conditions ${ }^{54}$ including childhood asthma ${ }^{55}$ and juvenile idiopathic arthritis, ${ }^{56}$ and compares with additional annual cost burdens reported elsewhere of $£ 890$ (1996-7 prices) for childhood depression, ${ }^{16} € 2748$ (2003 prices) for separation anxiety ${ }^{17}$ and between US\$1100 and 1800 (1996 prices) for ADHD. ${ }^{18}$ The study also revealed mean differences in the HUI3 and UK HUI2 multi-attribute utility scores of 0.192 and 0.118 , respectively, across the pooled study population between these comparison groups, which far exceeds the 0.03 minimally important difference in utility score postulated in the literature as clinically important for evaluative purposes. ${ }^{57}$ Notably, the difference in the mean HUI3 multi-attribute utility scores between children with (0.698) and without (0.890) a psychiatric disorder can be interpreted as a difference between being in a state of severe overall disability rather than a mild overall disability according to the classification of HUI3 multi-attribute utility scores published by the HUI developers. ${ }^{58}$

\section{Strengths and limitations}

The study population was drawn from participants in the EPICure study, a whole-population longitudinal study of all infants born extremely preterm in the UK and Republic of Ireland over a 10-month period and a contemporaneous classroom control group born at full term and matched for age, gender and ethnic group. As such, the study population consists of two distinct groups of children: one that can be characterised as at high risk for psychiatric disorders and a more representative general population sample. The two groups were analysed separately for the purposes of our empirical investigation. Of particular note 


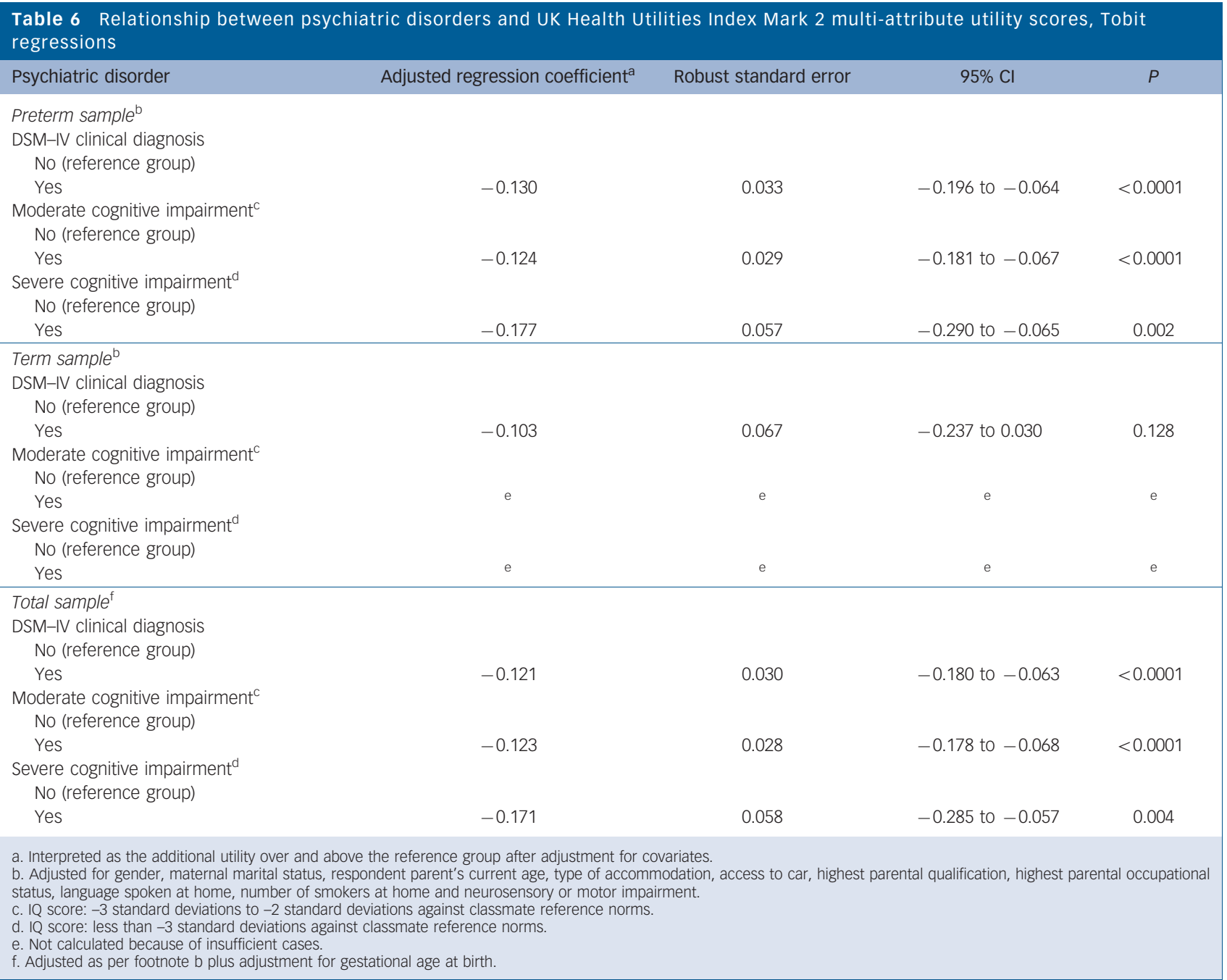

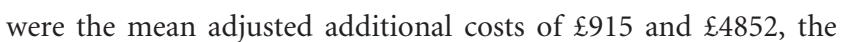
mean adjusted HUI 3 utility decrements of 0.205 and 0.342 and the mean adjusted UK HUI2 utility decrements of 0.124 and 0.177 associated with cognitive impairment and severe cognitive impairment, respectively, in the extremely preterm children. Analyses of the term-born children were restricted by the limited number of children in this population who were diagnosed with a psychiatric disorder $(n=11)$. Nevertheless, separate economic results for this population are presented for completeness. Additionally, analyses of the pooled study population controlled for clinical and sociodemographic confounders, including gestational age at birth and, alternatively, either a measure of neurosensory or motor impairment or an interaction term between gestational age at birth and psychiatric disorder. Consequently, we adopted a strategy that disentangled the effects of psychiatric disorders on economic outcomes from those that might be attributable to comorbidities. The study has a number of other strengths. Multi-informant psychiatric data were collected using the DAWBA for all children, rather than a subset identified at high risk, and diagnoses were made by consensus between two expert clinical raters who were masked to group allocation. The DAWBA has excellent reliability and validity ${ }^{28}$ and was the principal measure of psychopathology in the British mental health surveys. ${ }^{59}$ Cognitive ability was assessed by psychologists who achieved $\geqslant 95 \%$ interrater reliability across standardised tests, and neurosensory function was evaluated by experienced paediatricians. As loss to follow-up was more common among high-risk children, multiple imputation was used to estimate psychiatric diagnoses for 10 of the 331 study children for whom multi-informant psychiatric diagnoses were not performed. ${ }^{25}$ Other strengths of the study include validated and reliable approaches to measuring and valuing costs and preference-based health-related quality of life outcomes during childhood and a comprehensive analytical strategy.

There are a number of caveats to the study findings. First, the children included in the EPICure study, but excluded from our analyses because of loss to follow-up, were more likely to have had lower cognitive scores or cognitive impairment at 2.5 and 6 years. This suggests that we might have underestimated the true extent of psychiatric disorders in the study population. Second, the study population was too small to present cost and utility estimates at a granulated level for all childhood psychiatric disorders. A number of disorders, such as panic disorder, agoraphobia, obsessive-compulsive disorder, elective mutism, disinhibited attachment disorder of childhood, reactive attachment disorder, eating disorder, schizophrenia, manic episodes, ADHD hyperactive-impulsive subtype and Asperger syndrome, were not diagnosed in our study population. In addition, individual disorders had to be grouped into relatively broad categories, which might reflect disparate experiences in terms of resource utilisation and health-related quality of life. A 
much larger study population would be required to estimate costs and health utilities with sufficient statistical power for all childhood psychiatric disorders. Indeed, McClellan and colleagues have argued that a longitudinal study of 10000 children, consisting of 5000 characterised as at high risk of neurodevelopmental disabilities and 5000 randomly selected from the entire childhood population, is required to generate subtle information for the broad spectrum of conditions. ${ }^{60} \mathrm{~A}$ third caveat to the study findings is that the analysis of cost differences was conducted from a public sector perspective and encompassed costs of health, social and education services. It is likely that many psychiatric disorders have an impact on other sectors of the economy and on families and carers, ${ }^{16}$ suggesting that adopting a broader perspective would increase the cost differences between the study groups. A fourth caveat is that our cost estimates are based on parental reports of their child's resource utilisation over the previous year of life. Previous research has indicated that parents accurately recall their child's hospital service utilisation over extended periods when validated against medical records, but tend to underreport their child's community service utilisation. ${ }^{61}$ If this were the case for our study our absolute costs for community service utilisation may be underestimates. A fifth caveat is that, given the large number of children in our study with serious cognitive impairment and learning difficulties, the main parent rather than the child was considered the appropriate person to complete the HUI. Empirical evidence of the concordance between child and parent ratings of attributes of children's health-related quality of life suggests that parents are able to accurately rate observable behaviours, such as physical functioning and physical symptoms, but are less successful at identifying social or emotional impairments. ${ }^{62,63}$ However, there is no consistent evidence to suggest that parents consistently either underreport or overreport social or emotional impairments, ${ }^{64}$ which suggests that there are unlikely to be systematic biases in the measurement of health-related quality of life in our study. A final caveat is that the underlying preference weights for the HUI3 and UK HUI2 multi-attribute utility measures have been derived from surveys of adults rather than of children. ${ }^{4-46}$ The cognitive requirements entailed in directly estimating preference weights among our study population, many of whom had developmental disabilities, was considered too burdensome. Nevertheless, our approach of valuing health outcomes using population-based preferences is in line with the recommendations of many decision-making bodies, such as the National Institute for Health and Clinical Excellence in England and Wales. ${ }^{36}$

\section{Implications}

How might the results of our study be used? Given recent evidence of the increasing incidence of some childhood psychiatric disorders, ${ }^{60}$ it is imperative that clinical decision-makers and budgetary and service planners recognise the overall economic impact of each condition in their service planning, as well as the potential contribution of clinical and sociodemographic factors to economic outcomes. More pertinently, in our opinion, our mean cost and utility estimates and their associated distributions can act as data inputs for cost-effectiveness models of preventive or treatment interventions for childhood psychiatric disorders. Economic analysts who construct decision-analytic models are often faced with estimating costs and health utilities for a large number of health conditions or states with limited resources or time. Under these circumstances our catalogue can be viewed as a significant new resource that can act as data inputs or be pooled with the totality of the existing evidence base. It should be noted, however, that analysts may face a particular methodological challenge when the time horizon for the cost-effectiveness model spans the entire period of childhood or further into adulthood. Under these circumstances, the impact of age on costs and health utilities should be estimated from data gathered in large-scale longitudinal studies as they become available. When such data are not available, techniques such as meta-regression of data across a number of studies should be considered as a means of disentangling the impact of age.

\footnotetext{
Stavros Petrou, PhD, Warwick Clinical Trials Unit, University of Warwick, Coventry; Samantha Johnson, PhD, Division of Academic Neonatology, Institute for Women's Health, University College London, London; Dieter Wolke, PhD, Department of Psychology and Health Science Research Institute, University of Warwick, Coventry: Chris Hollis, MRCPsych, Puja Kochhar, BM, Division of Psychiatry, School of Community Health Sciences, University of Nottingham, Nottingham; Neil Marlow, Fmedsci, Division of Academic Neonatology, Institute for Women's Health, University College London, London, UK

Correspondence: Stavros Petrou, PhD, Warwick Clinical Trials Unit, University of Warwick, Coventry CV4 7AL, UK. Email: S.Petrou@warwick.ac.uk

First received 12 Apr 2010, final revision 6 July 2010, accepted 21 Jul 2010
}

\section{Funding}

The EPICure Study was funded by the Medical Research Council, UK. S.P. was funded by a MRC Senior Non-Clinical Research Fellowship during the course of this study.

\section{Acknowledgements}

We are indebted to the EPICure Study Group, which includes paediatricians in 276 maternity units in the UK and Republic of Ireland who identified the original cohort, contributed perinatal data and whose help was invaluable. We would also like to thank the children who participated in the EPICure Study and the parents who completed the relevant research instruments.

\section{References}

1 Roberts R. Prevalence of psychopathology among children and adolescents. Am J Psychiatry 1998; 155: 715-25.

2 Meltzer H, Gatward R, Goodman R, Ford T. Mental health of children and adolescents in Great Britain. Int Rev Psychiatry 2003; 15: 185-7.

3 Costello EJ, Mustillo S, Erkanli A, Keeler G, Angold A. Prevalence and development of psychiatric disorders in childhood and adolescence. Arch Gen Psychiatry 2003; 60: 837-44.

4 Kovacs M, Feinberg TL, Crouse-Novak M, Palauskas SL, Finkelstein R. Depressive disorders in childhood: I. A longitudinal prospective study of characteristics and recovery. Arch Gen Psychiatry 1984; 41: 229-37.

5 Kovacs M, Feinberg TL, Crouse-Novak M, Palauskas SL, Pollack M, Finkelstein R. Depressive disorders in childhood: II. A longitudinal study of the risk for a subsequent major depression. Arch Gen Psychiatry 1984; 41: 643-9.

6 Weiss G, Hechtman L, Milroy T, Perlman T. Psychiatric status of hyperactives as adults: a controlled prospective 15-year follow-up of 63 hyperactive children. J Am Acad Child Psychiatry 1985; 24: 211-20.

7 Lambert N. Adolescent outcomes for hyperactive children. Am Psychol 1988; 43: 786-99.

8 Keller MB, Lavori PW, Wunder J, Beardslee WR, Schwartz CE, Roth J. Chronic course of anxiety disorders in children and adolescents. J Am Acad Child Psychiatry 1992; 31: 595-99.

9 Cohen P, Cohen J, Brook J. An epidemiological study of disorders in late childhood and adolescence: II. Persistence of disorders. J Child Psychol Psychiatry 1993; 34: 869-77.

10 Cohen P, Cohen J, Kasen S, Velez CN, Hartmarh C, Johnson J, et al. An epidemiological study of disorders in late childhood and adolescence: I. Ageand gender-specific prevalence. J Child Psychol Psychiatry 1993; 34: 851-67.

11 Fergusson DM, Horwood LJ, Lynskey MT. Prevalence and comorbidity of DSM-III-R diagnoses in a birth cohort of 15 year olds. J Am Acad Child Psychiatry 1993; 32: 1127-34.

12 Loeber R, Green S, Keenan K, Lahey BB. Which boys will fare worse? Early predictors of the onset of conduct disorder in a six-year longitudinal study. $J$ Am Acad Child Psychiatry 1995; 34: 499-509. 
13 Mannuzza S, Klein RG, Bessler A, Malloy P, LaPadula M. Adult psychiatric status of hyperactive boys grown up. Am J Psychiatry 1998; 155: 493-8.

14 Biederman J, Monuteaux MC, Mick E, Spencer T, Wilens TE, Silva JM, et al. Young adult outcome of attention deficit hyperactivity disorder: a controlled 10-year follow-up study. Psychol Med 2006; 36: 167-79.

15 Giaconia RM, Reinberg HZ, Silverman AB, Pakiz B, Frost AK, Cohen E. Age on onset of psychiatric disorders in a community population of older adolescents. J Am Acad Child Psychiatry 1994; 33: 706-17.

16 Mccrone $\mathrm{P}$, Knapp M, Fombonne E. The Maudsley long-term follow-up of child and adolescent depression: predicting costs in adulthood. Eur Child Adolesc Psychiatry 2005; 14: 407-13.

17 Bodden DH, Dirksen CD, Bögels SM. Societal burden of clinically anxious youth referred for treatment: a cost-of-illness study. J Abnorm Child Psychol 2008; 36: 487-97.

18 Leibson $\mathrm{CL}$, Long $\mathrm{KH}$. Economic implications of attention-deficit hyperactivity disorder for healthcare systems. Pharmacoeconomics 2003; 21: 1239-62.

19 Matza LS, Secnik K, Rentz AM, Mannix S, Sallee FR, Gilbert D, et al. Assessment of health state utilities for attention-deficit/hyperactivity disorder in children using parent proxy report. Qual Life Res 2005; 14 : 735-47

20 Petrou S, Kupek E. Estimating preference-based Health Utilities Index Mark 3 utility scores for childhood conditions in England and Scotland. Med Decis Making 2009; 29: 291-303.

21 Wood NS, Marlow N, Costeloe K, Gibson AT, Wilkinson AR for the EPICure Study Group. Neurologic and developmental disability after extremely preterm birth. New Engl J Med 2000; 343: 378-84

22 Botting N, Powls A, Cooke R, Marlow N. Attention deficit hyperactivity disorder and other psychiatric outcomes in very low birthweight children at 12 years. J Child Psychol Psychiatry 1997; 38: 931-41.

23 Elgen I, Sommerfelt K, Markestad T. Population based, controlled study of behavioural problems and psychiatric disorders in low birthweight children at 11 years of age. Arch Dis Child Fetal Neonatal Ed 2002; 87: F128-32.

24 Indredavik MS, Vik T, Heyerdahl S, Kulseng S, Fayers P, Brubakk AM. Psychiatric symptoms and disorders in adolescents with low birth weight. Arch Dis Child Fetal Neonatal Ed 2004; 89: F445-50.

25 Johnson S, Hollis C, Kochhar P, Hennessy E, Wolke D, Marlow N. Psychiatric disorders in extremely preterm children: longitudinal finding at age 11 years in the EPICure study. J Am Acad Child Adolesc Psychiatry 2010; 49: 453-63.

26 Marlow N, Wolke D, Bracewell MA, Samara M for the EPICure Study Group. Neurologic and developmental disability at six years of age after extremely preterm birth. New Engl J Med 2005; 352: 9-19.

27 Johnson S, Hennessy EM, Smith R, Trikic R, Wolke D, Marlow N. Academic attainment and special educational needs in extremely preterm children at 11 years of age: the EPICure Study. Arch Dis Child Fetal Neonatal Ed 2009 94: F283-9.

28 Goodman R, Ford T, Richards H, Gatward R, Meltzer H. The Development and Well-Being Assessment: description and initial validation of an integrated assessment of child and adolescent psychopathology. J Child Psychol Psychiatry 2000; 41: 645-55.

29 World Health Organization. The ICD-10 Classification of Mental and Behavioural Disorders. World Health Organization, 1992

30 American Psychiatric Association. Diagnostic and Statistical Manual of Mental Disorders (4th edn) (DSM-IV). APA, 1994.

31 American Psychiatric Association. Diagnostic and Statistical Manual of Mental Disorders (4th edn) Text Revision (DSM-IV-TR). APA, 2000.

32 Kaufman AS, Kaufman NL. Kaufman Assessment Battery for Children. American Guidance Service, 1983

33 Wolke D, Ratschinski G, Ohrt B, Riegel K. The cognitive outcome of very preterm infants may be poorer than often reported: an empirical investigation of how methodological issues make a big difference. Eur J Pediatr 1994; 153: 906-15.

34 Allen C, Beecham J. Costing services: ideals and reality. In Costing Community Care: Theory and Practice (eds A Netten, J Beecham): 25-42. Ashgate Publishing, 1993.

35 Drummond MF, Sculpher MJ, Torrance GW, O'Brien BJ, Stoddart GL. Methods for the Economic Evaluation of Health Care Programmes, 3rd edn. Oxford University Press, 2005.

36 National Institute for Health and Clinical Excellence. Guide to the Methods of Technology Appraisal. NICE, 2008.
37 Department of Health, England. NHS Reference Costs 2006-07. The Information Centre, Department of Health, 2007.

38 Curtis L. Unit Costs of Health and Social Care. Personal Social Services Research Unit (PSSRU), University of Kent, 2007.

39 Paediatric Formulary Committee. BNF for Children, 2007. BMJ Publishing Group, Pharmaceutical Press, and RCPCH Publications, 2007.

40 Torrance GW, Furlong W, Feeny D, Boyle M. Multi-attribute preference functions: Health Utilities Index. Pharmacoeconomics 1995; 7: 503-20.

41 Eiser C, Morse R. Quality-of-life measures in chronic diseases of childhood. Health Technol Assess 2001; 5: 1-156.

42 Furlong WJ, Feeny DH, Torrance GW, Barr RD. The Health Utilities Index (HUI) system for assessing health-related quality of life in clinical studies. Ann Med 2001; 33: 375-84.

43 Horsman J, Furlong W, Feeny D, Torrance GW. The Health Utilities Index (HUI): concepts, measurement properties and applications. Health Qual Life Outcomes 2003; 1: 54.

44 Feeny D, Furlong W, Torrance GW, Goldsmith $\mathrm{CH}$, Zhu Z, DePauw S, et al. Multiattribute and single-attribute utility functions for the Health Utilities Index Mark 3 system. Med Care 2002; 40: 113-28.

45 Furlong W, Feeny D, Torrance GW, Goldsmith $\mathrm{CH}$, DePauw S, Zhu Z, et al. Multiplicative Multi-attribute Utility Function for the Health Utilities Index Mark 3 (HUI3) System: A Technical Report. Working Paper 98-11. Centre for Health Economics and Policy Analysis, McMaster University, Canada, 1998.

46 McCabe C, Stevens K, Roberts J, Brazier J. Health state values for the HUI 2 descriptive system: results from a UK survey. Health Econ 2005; 14: 231-44.

47 Schafer JL. Multiple imputation: a primer. Stat Methods Med Res 1999; 8: 3-15.

48 Briggs A, Clark T, Wolstenholme J, Clarke P. Missing . . . presumed at random: cost-analysis of incomplete data. Health Econ 2003; 12: 377-92.

49 Gray A, Wolstenholme J, Wordsworth S, Clarke P. Applied Methods of Cost-Effectiveness Analysis. Oxford University Press, 2010.

50 Barber JA, Thompson SG. Analysis of cost data in randomized trials: an application of the non-parametric bootstrap. Stat Med 2000; 19: 219-36.

51 Briggs A, Claxton K, Sculpher M. Decision Modelling for Health Economic Evaluation. Oxford University Press, 2006.

52 Johnson S, Fawke J, Hennessy E, Rowell V, Thomas S, Wolke D, et al. Neurodevelopmental disability through 11 years of age in children born before 26 weeks of gestation. Pediatrics 2009; 124: e249-57.

53 Greene WH. Econometric Analysis, 5th edn. Prentice Hall, 2003.

54 Ungar W. Economic Evaluation in Child Health. Oxford University Press, 2009

55 To T, Dell S, Dick P, Cicutto L. The burden of illness experienced by young children associated with asthma: a population-based cohort study. J Asthma 2008; 45: 45-9.

56 Thornton J, Lunt M, Ashcroft DM, Baildam E, Foster H, Davidson J, et al. Costing juvenile idiopathic arthritis: examining patient-based costs during the first year after diagnosis. Rheumatology 2008; 47: 985-90.

57 Drummond M. Introducing economic and quality of life measurements into Clinical studies. Ann Med 2001; 33: 344-9.

58 Feeny D, Furlong W, Saigal S, Sun J. Comparing directly measured standard gamble scores to HUI2 and HUI3 utility scores: group- and individual-level comparisons. Soc Sci Med 2004; 58: 799-809.

59 Green H, McGinnity A, Meltzer H, Ford T, Goodman R. Mental Health of Children and Young People in Great Britain, 2004. Office for National Statistics, 2004.

60 McClellan J, Bresnahan MA, Echeverria D, Knox SS, Susser E. Approaches to psychiatric assessment in epidemiological studies of children. J Epidemiol Community Health 2009; 63: i4-i14.

61 Petrou S, Murray L, Cooper P, Davidson LL. The accuracy of self-reported health care resource utilization in health economic studies. Int J Technol Assess Health Care 2002; 18: 705-10.

62 Petrou S. Methodological issues raised by preference-based approaches to measuring the health status of children. Health Econ 2003; 12: 697-702.

63 Verrips GHW, Stuifbergen MC, den Ouden LA, Bonsel GJ, Gemke RJ, Paneth $\mathrm{N}$, et al. Measuring health status using the Health Utility Index: agreement between raters and between modalities of administration. $J$ Clin Epidemiol 2001; 54: 475-81.

64 Eiser C, Morse R. Quality-of-life measures in chronic diseases of childhood Health Technol Assess 2001; 5: 1-156. 